\title{
Physical Properties of Wave Motion in Inclined Magnetic Fields within Sunspot Penumbrae
}

\author{
H. Schunker · D.C. Braun • C. Lindsey · P.S. Cally
}

Received: 6 October 2007 / Accepted: 6 February 2008 / Published online: 2 March 2008

(C) The Author(s) 2008. This article is published with open access at Springerlink.com

\begin{abstract}
At the surface of the Sun, acoustic waves appear to be affected by the presence of strong magnetic fields in active regions. We explore the possibility that the inclined magnetic field in sunspot penumbrae may convert primarily vertically-propagating acoustic waves into elliptical motion. We use helioseismic holography to measure the modulus and phase of the correlation between incoming acoustic waves and the local surface motion within two sunspots. These correlations are modeled by assuming the surface motion to be elliptical, and we explore the properties of the elliptical motion on the magnetic-field inclination. We also demonstrate that the phase shift of the outward-propagating waves is opposite to the phase shift of the inward-propagating waves in stronger, more vertical fields, but similar to the inward phase shifts in weaker, more-inclined fields.
\end{abstract}

Keywords Helioseismology $\cdot$ Observations $\cdot$ Sunspots $\cdot$ Penumbrae

\section{Introduction}

Helioseismology uses the observed solar-surface acoustic wave field to construct images of the subsurface structure of the Sun. Of particular interest has been the three-dimensional (3D) modeling of time-distance (Duvall et al., 1993) observations of travel-time shifts to

Helioseismology, Asteroseismology, and MHD Connections Guest Editors: Laurent Gizon and Paul Cally.

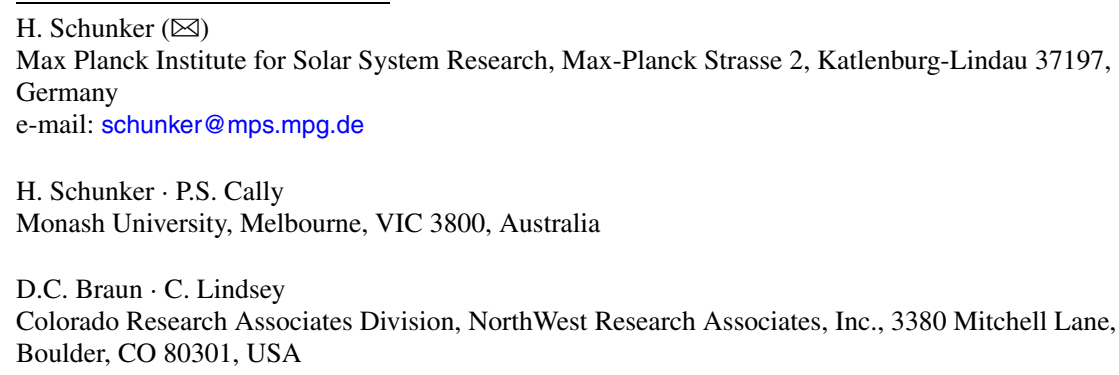


deduce the subsurface structure of active regions (Kosovichev, Duvall, and Scherrer, 2000; Zhao and Kosovichev, 2003). By assuming that the travel-time shifts are due to perturbations in the sound speed below the spot, a general consensus in the models has emerged consistent with sound-speed reductions (relative to the surrounding quiet Sun) near the surface $(\lesssim 4 \mathrm{Mm})$ and enhancements up to $15 \mathrm{Mm}$ below sunspots (Kosovichev, Duvall, and Scherrer, 2000; Couvidat, Birch, and Kosovichev, 2006). Using ring-diagram analysis, Basu, Antia, and Bogart (2004) also find a lower sound speed immediately below the surface and an increase in the sound speed below $7 \mathrm{Mm}$.

A comparison between Fourier-Hankel analysis and time-distance results by Braun (1997) first prompted caution in the interpretation of acoustic-oscillation signals within sunspots. The influences of strong surface magnetic fields have not been explicitly included in most helioseismic models of active regions. Lindsey and Braun (2005a, 2005b) have shown that helioseismic phase shifts observed with helioseismic holography vanish below a depth of about $5 \mathrm{Mm}$, when a surface phase shift ("showerglass") based on photospheric magnetic flux density, is removed from the data.

Other evidence supports the possibility of strong near-surface contributions to the helioseismic phase (or travel-time) shifts. These include the possible contamination of 3D inversions by surface perturbations (e.g., Korzennik, 2006; Couvidat and Rajaguru, 2007). It has also been shown that the reduction of $p$-mode amplitudes in magnetic regions can cause travel-time shifts (Rajaguru et al., 2006). The suppression of sources of wave excitation within sunspots can also produce measurable shifts (Hanasoge et al., 2007).

Schunker et al. (2005) and Schunker, Braun, and Cally (2007) have found that phase shifts obtained from seismic holography in sunspot penumbrae vary with the line-of-sight angle (from vertical) as projected onto the plane containing the magnetic field and the vertical direction. A similar effect has also been noted by Zhao and Kosovichev (2006) with time-distance measurements. Schunker, Braun, and Cally (2007) find that the effect is dependent upon the strength and/or inclination of the magnetic field. In the penumbra, the magnetic field strength decreases as the magnetic field angle from vertical increases; hence the two properties of the magnetic field cannot be extricated. The phase variation with lineof-sight viewing angle is demonstrated most substantially at frequencies around $5 \mathrm{mHz}$, with a strong, almost vertical magnetic field close to the umbra. Schunker, Braun, and Cally (2007) also find that the total variation across all lines of sight increases with temporal frequency, particularly in the stronger fields in the penumbrae.

Mode conversion of the acoustic waves in the near surface has been explored, in two dimensions, as the physical cause of the observed absorption of acoustic waves by sunspots. A fast acoustic wave, propagating toward the surface from the interior, encounters the depth at which the Alfvén speed is equal to the sound speed $(a \approx c)$, which is typically close to the surface in a sunspot. Under these conditions, it is able to transmit to a slow acoustic mode and convert to a fast magnetic mode (Cally, 2005). Crouch and Cally (2003) explore the mode conversion in two dimensions with a uniform inclined magnetic field relevant to sunspot penumbrae. The inclination of the magnetic field is found to have a significant effect on the likelihood of conversion and fits extremely well with the analysis of Braun (1995), as shown by Cally, Crouch, and Braun (2003). Further work (Cally, 2005; Schunker and Cally, 2006) using ray theory has since established that it is the angle between the acoustic wave path and the magnetic field (the "attack angle") that is the crucial factor inducing conversion. With large attack angle at the $a \approx c$ level there is maximum conversion from a fast-acoustic mode to a fast-magnetic mode. A small attack angle encourages transmission to a slowacoustic mode, which is guided "up" the magnetic field lines to observational heights in the atmosphere. A consequence of mode conversion may be the observational signature of elliptical motion in regions of inclined magnetic field. 
The aim of this paper is to model the observations of two sunspots previously analyzed by Schunker, Braun, and Cally (2007) to determine the properties of velocity ellipses consistent with the data. These models are based on a least-squares fit of the phase and modulus information of the local ingression control correlation. We explore the properties of these ellipses, observed with waves of different temporal frequencies, as functions of the magnetic field inclination angle. We also examine the variation with line-of-sight angle of the phase shifts in the outgoing waves, using the local egression control correlation, to assess the relation of the phase-shift variations between incoming and outgoing waves.

In the following sections we describe the data (Section 2), give an outline of the helioseismic-holography technique used (Section 3), describe the results (Section 4), and discuss our results in the context of mode conversion (Section 5).

\section{Observations}

As this is a continuation of studies by Schunker et al. (2005) and Schunker, Braun, and Cally (2007) we use the same data; however, we provide a short description here to maintain coherence. The Michelson Doppler Imager (MDI) onboard the Solar and Heliospheric Observatory (SOHO) (Scherrer et al., 1995) provides the solar-surface Doppler-velocity information. The Dopplergrams are full disk, have a 60 -second cadence, and have a resolution of $\approx 1.4 \mathrm{Mm}$ per pixel. These full-disk Dopplergrams are Postel projected and a $512 \times 512$ pixel area is extracted centered on the active region. We analyze two sunspots. The first, in AR9026, was observed over ten days from 3 to 12 June 2000 with a Carrington longitude $\left(L_{0}\right)$ of $75^{\circ}$ and latitude $\left(B_{0}\right)$ of $20^{\circ}$ and penumbral boundaries defined by inner and outer radii of $7 \mathrm{Mm}$ and $16 \mathrm{Mm}$, respectively. The second is a sunspot in AR9057 observed over nine days from 24 June to 2 July 2000 with $L_{0}=158^{\circ}$ and $B_{0}=13^{\circ}$ and inner and outer penumbral boundaries given by $6 \mathrm{Mm}$ and $13 \mathrm{Mm}$, respectively. The penumbral boundaries are defined to be between $50 \%$ and $85 \%$ of the nearby quiet-Sun continuum intensity. The sunspots in these active regions were chosen based on the existence of continuous MDI observation as they traversed the solar disk, and for their relatively simple magnetic structure and evolution.

Magnetograms from the Imaging Vector Magnetograph (IVM) at the University of Hawaii Mees Solar Observatory (Mickey et al., 1996) provide the orientation and strength of the surface magnetic field in the sunspots chosen in AR9026 and AR9057. The IVM observations are made over a 28-minute interval: for AR9026 starting at 18:29 Universal Time (UT) on 5 June 2000 and for AR9057 starting at 16:19 UT on 28 June 2000. The IVM data reveal an azimuthally spreading magnetic-field configuration for both sunspots although they are not entirely symmetric. It is assumed, supported largely by available lineof-sight magnetograms, that there is no significant evolution of the magnetic field in the sunspots during the time of observation. Therefore, using only one vector magnetogram for the duration of the observation is reasonable. Rotation and scaling are applied to align the IVM data to the line-of-sight MDI magnetograms.

Figure 1 shows a strong correlation between the magnetic field inclination (from vertical, $\gamma$ ) and field strength (with the strong field being almost vertical whereas the highly inclined field is relatively weak). In this paper we use the inclination $\gamma$ as the primary variable, dividing the penumbrae into three regions defined by the values of $\gamma$ (Figure 1). It is understood in this analysis that the magnetic field strength is implicitly correlated with the inclination through Figure 1 and that independent dependencies of observables with field strength and inclination are not extracted. The dependence of the phase shifts on the line-of-sight viewing angle of the magnetic field is facilitated by knowing the full vector magnetic field. 


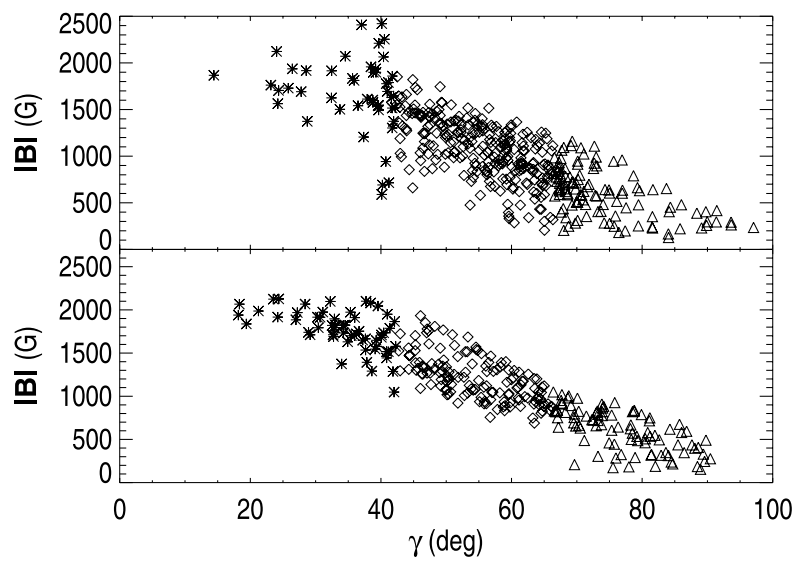

Figure 1 Magnetic field strength $(|B|)$ plotted against inclination from vertical $(\gamma)$ determined from IVM vector magnetograms for AR9026 (5 June 2000) (bottom) and AR9057 (28 June 2000) (top). The different symbols divide the penumbra into roughly equal regions of inclination: $\gamma<42^{\circ}$ (asterisks); $42^{\circ}<\gamma<66^{\circ}$ (diamonds); $\gamma>66^{\circ}$ (triangles). This corresponds to average magnetic field strengths of 1700, 1000, and $600 \mathrm{G}$ for AR9057 and 1900, 1400, and $600 \mathrm{G}$ for AR9026. In general, the progression from the upper left portion of the distribution to the lower right portion represents increasing distance from the center of the spot.

\section{Helioseismic Holography}

Helioseismic holography (Lindsey and Braun, 2000; Braun and Lindsey, 2000) is the phasecoherent imaging of the solar subsurface based on photospheric acoustic oscillations. The ingression is an assessment of the observed wave field $\left[\psi\left(\mathbf{r}^{\prime}, t\right)\right]$ converging to a selected focal point $(\mathbf{r}, z, t)$ and the egression is the time reverse (an assessment of waves diverging from that point). In this case, we calculate the quantities at the surface, $z=0$. In practice, the observed wave field used for the calculation is usually an annulus surrounding the chosen focal point. The pupil used here is identical to that described by Schunker et al. (2005) and is constructed for the calculations with inner radius $a=20.7 \mathrm{Mm}$ and outer radius $b=43.5 \mathrm{Mm}$, designed to be large enough that when the focal point is within the penumbra the area covered by the annulus does not include large areas of strong magnetic field. At a frequency of $5 \mathrm{mHz}$ this selects $p$ modes with spherical harmonic degree and radial degree between $\ell \approx 450$ and $\ell \approx 700$. The ingression at the surface is given by

$$
H_{-}(\mathbf{r}, 0, t)=\int_{a<\left|\mathbf{r}-\mathbf{r}^{\prime}\right|<b} \mathrm{~d}^{2} \mathbf{r}^{\prime} G_{-}\left(\left|\mathbf{r}-\mathbf{r}^{\prime}\right|, 0, t-t^{\prime}\right) \psi\left(\mathbf{r}^{\prime}, t\right)
$$

where $G_{-}$is the ingression Green's function (Lindsey and Braun, 2000). The egression $\left(H_{+}\right)$ is simply the time reverse of Equation (1) (i.e., $t^{\prime}-t$ ).

The ingression (1) is correlated with the observed wave field in the space-frequency domain,

$$
C_{-}(\mathbf{r}, v)=\left\langle\left.\hat{H}_{-}(\mathbf{r}, v) \hat{\psi}^{*}(\mathbf{r}, v)\right|_{\Delta v}=\left|C_{-}\right| \mathrm{e}^{-\mathrm{i} \delta \phi_{-}},\right.
$$

where $\hat{H}_{-}(\mathbf{r}, v)$ and $\hat{\psi}(\mathbf{r}, v)$ are the temporal Fourier transforms of $H_{-}$and $\psi$, respectively, and we have dropped the dependence on depth $(z)$. The correlation has a modulus $\left|C_{-}\right|$ and a phase $\delta \phi_{-}$. In the frequency spectrum, with the pupil covering mostly quiet Sun, the local ingression control correlation simply characterizes how the local magnetic photosphere responds acoustically to upcoming waves, prescribed by $H_{-}$, originating within the pupil. 
In Schunker et al. (2005) and Schunker, Braun, and Cally (2007) the phase shift, caused by the surface perturbations to the incident wave, is calculated at various frequencies. Here we extend that research and monitor the variation of the correlation amplitude within the sunspot penumbral region, which is then combined with the phase information to form estimates of the surface velocity ellipse. We then go on to examine the phase of the local egression control correlation $\left[\delta \phi_{+}=\operatorname{Arg}\left[C_{+}(\mathbf{r}, v)\right]\right]$ with the line-of-sight angle in the penumbra of sunspots in AR9057 and AR9026.

\section{Elliptical Representation of Surface Doppler Signals}

For better statistics, multiple days of observation of each sunspot are combined and a leastsquares fit of the observations allows an estimation of the velocity ellipse. To calculate the velocity ellipse, the ingression correlation (Equation (2)) is used as a proxy for the local velocity. The modulus and phase of the correlation vary with respect to the line-of-sight angle and these variations are modeled to construct an ellipse representing the surface velocity associated with a particular magnetic field element.

A smeared ingression "flat field" takes out undesired contributions to the correlation modulus owing to the temporal or spatial variations of the ingression and the presence of magnetic regions in the pupil. This is achieved by dividing $\left|C_{-}\right|$by the Gaussian smear of the root-mean square of the ingression to get $\left|C_{-}\right|_{\text {flat }}$. The resulting correlations are normalized so that the ingression correlation modulus in the nearby quiet Sun has a value of $\cos (\zeta)$, where $\zeta$ is the heliocentric angle of the quiet region from disk center. A dependence of the quiet Sun (root-mean-squared) amplitude with $\cos (\zeta)$ is expected for predominantly vertically oscillating $p$ modes. The normalization is achieved by dividing $\left|C_{-}\right|_{\text {flat }}$ by the quiet-Sun average divided by the cosine of the heliocentric angle, that is,

$$
\left|C_{-}\right|_{\text {norm }}=\left|C_{-}\right|_{\text {flat }} \frac{\cos (\zeta)}{\left\langle\left|C_{-}\right|\right\rangle_{\mathrm{QS}}}
$$

The normalization corrects for variations in the modulus from duty-cycle variations, foreshortening, and other effects that cause undesired variations in the modulus from day to day. These procedures assume that these factors have the same relative effect on the (desired) correlations in the penumbra as they do to the quiet Sun. It is difficult to assess the validity of this, and so it is used as a reasonable working assumption subject to some caution.

We use the same angle $\theta_{\mathrm{p}}$ as defined in Schunker et al. (2005). The line-of-sight vector is projected onto the plane containing the local magnetic field vector and the radial vector. We define $\theta_{\mathrm{p}}$ as the angle between this projected line-of-sight vector and the radial vector. We now simply refer to $\left|C_{-}\right|_{\text {norm }}$ as $\left|C_{-}\right|$and see how it varies with $\theta_{\mathrm{p}}$.

Figures 2, 3, 4 and 6, 7, 8 show the variation of $\left|C_{-}\right|$with $\theta_{\mathrm{p}}$ for AR9026 and AR9057 in the left columns at 3,4 , and $5 \mathrm{mHz}$ in the same three bins of inclination shown in Figure 1. The right column shows $\delta \phi_{-}$. The variations of $\delta \phi_{-}$with $\theta_{\mathrm{p}}$ have been the subject of our previous analyses (Schunker et al., 2005; Schunker, Braun, and Cally, 2007). In the absence of magnetic effects, we expect the local oscillatory wave field, as assessed by the ingression correlation, to be consistent with purely vertical motion. This would predict a dependence of $\left|C_{-}\right|$on $\cos \left(\theta_{\mathrm{p}}\right)$ and no dependence of $\delta \phi_{-}$on $\cos \left(\theta_{\mathrm{p}}\right)$. Departures from these expectation are clearly visible in Figures 2-4 and 6-8. A rough understanding of these results can be gained by noting that the net variation in the phase shift with $\theta_{\mathrm{p}}$ is related to the eccentricity of the ellipse, whereas the value of $\theta_{\mathrm{p}}$ for maximum $\left|C_{-}\right|$determines the orientation of the 


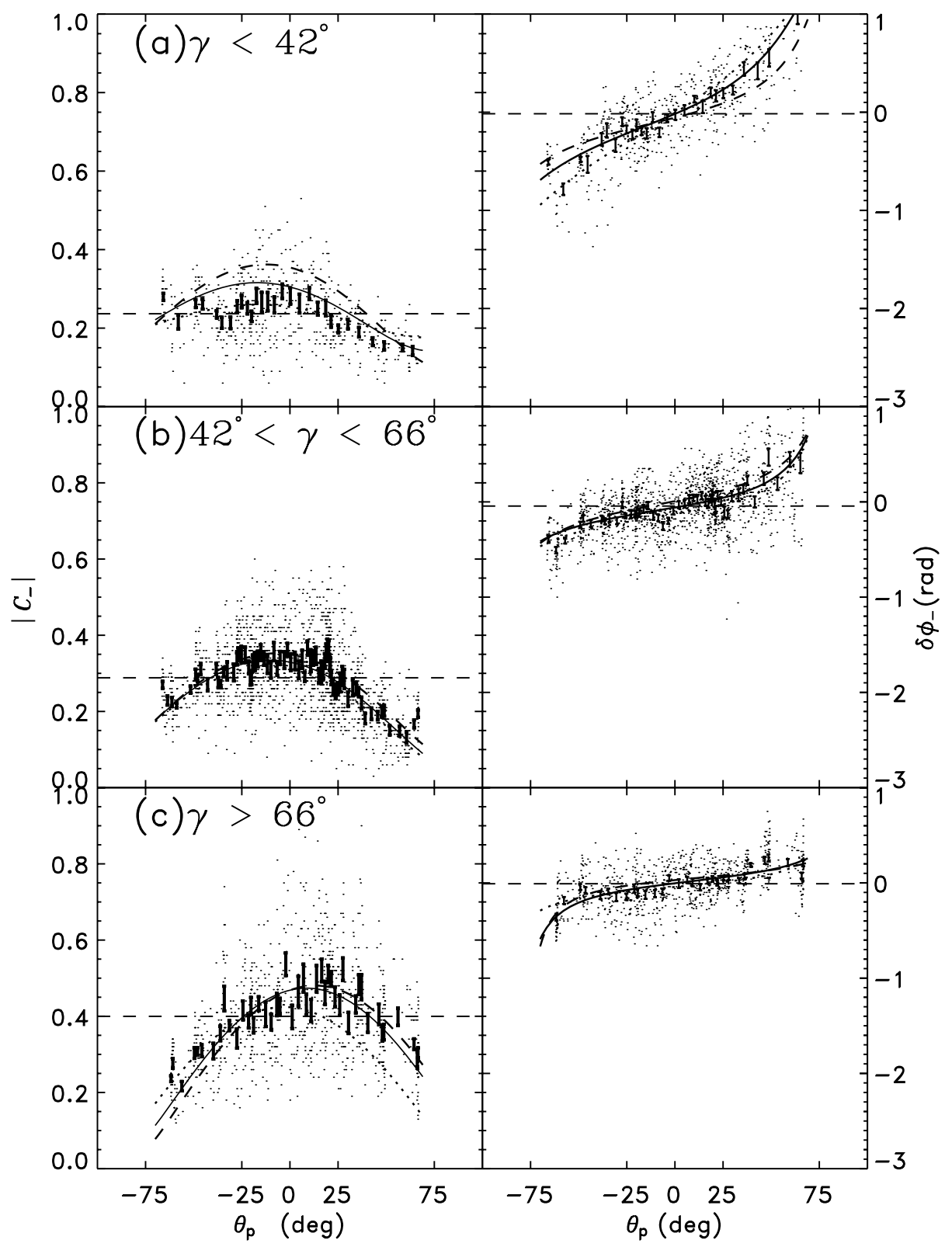

Figure 2 The modulus of the correlation, $\left|C_{-}\right|$(left column), and the phase $\delta \phi_{-}$(right column) in the penumbra of AR9026 at $3 \mathrm{mHz}$ for all days of observation plotted against projected angle $\theta_{\mathrm{p}}$ for different values of magnetic field inclinations as indicated: (a) $\gamma<42^{\circ}$, where the mean field strength is $\langle\mathbf{B}\rangle=1900 \mathrm{G}$; (b) $42^{\circ}<\gamma<66^{\circ}$, where $\langle\mathbf{B}\rangle=1400 \mathrm{G}$; (c) $\gamma>66^{\circ}$, where $\langle\mathbf{B}\rangle=600 \mathrm{G}$. The horizontal dashed lines indicate the mean value of $\left|C_{-}\right|$for each panel. The error bars indicate the standard deviation of the mean over bins of 20 measurements in $\theta_{\mathrm{p}}$. The solid line is a fit for all the displayed data; the dotted line is a fit for the data from 3 to 7 June 2000; the dashed line is a fit for data from 8 to 12 June 2000. 


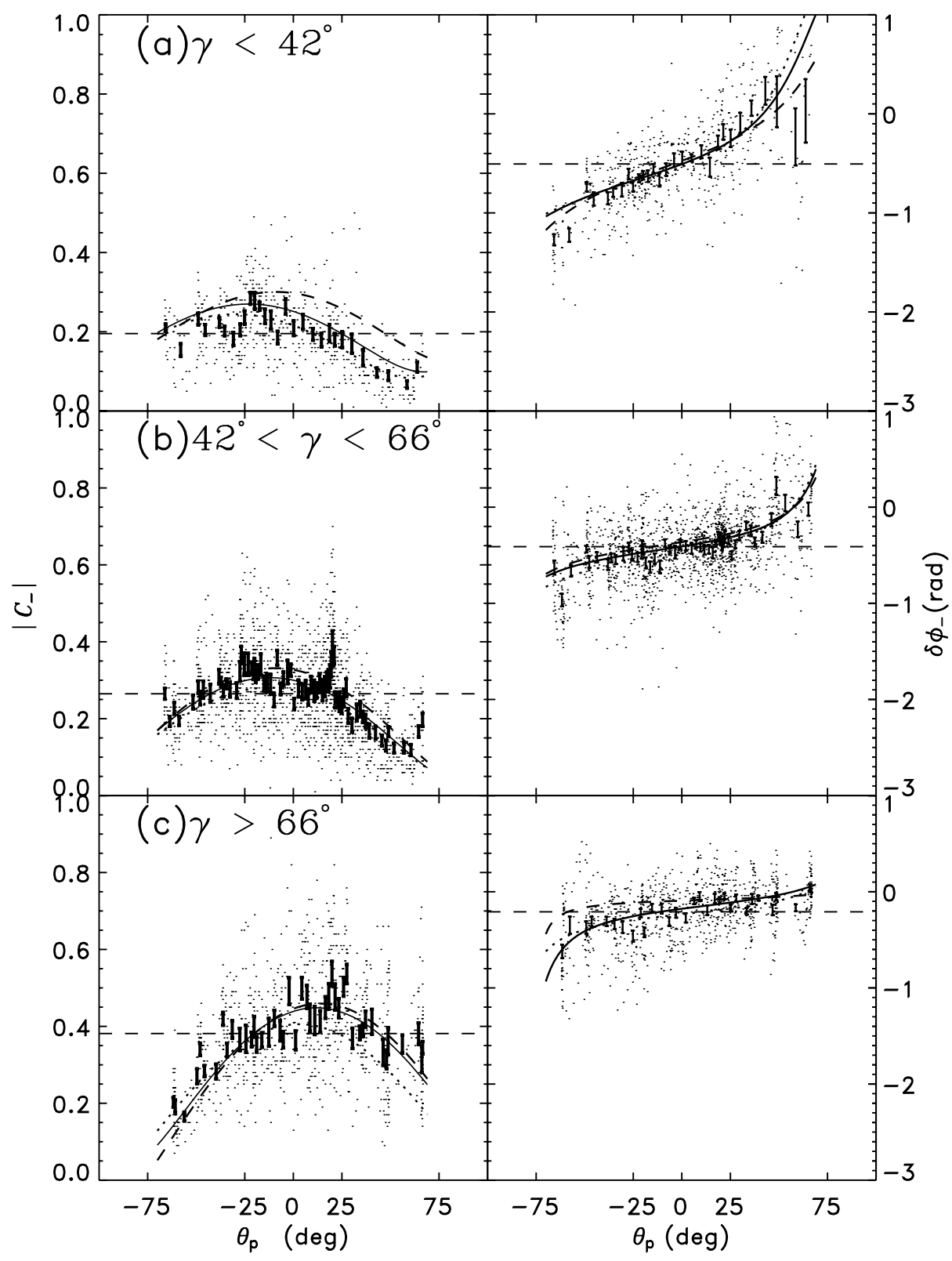

Figure 3 The modulus of the correlation, $\left|C_{-}\right|$(left column), and the phase $\delta \phi_{-}$(right column) in the penumbra of AR9026 at $4 \mathrm{mHz}$ for all days of observation plotted against projected angle $\theta_{\mathrm{p}}$ for different values of magnetic field inclinations as indicated: (a) $\gamma<42^{\circ}$, where the mean field strength is $\langle\mathbf{B}\rangle=1900 \mathrm{G}$; (b) $42^{\circ}<\gamma<66^{\circ}$, where $\langle\mathbf{B}\rangle=1400 \mathrm{G}$; (c) $\gamma>66^{\circ}$, where $\langle\mathbf{B}\rangle=600 \mathrm{G}$. The horizontal dashed lines indicate the mean value of $\left|C_{-}\right|$for each panel. The error bars indicate the standard deviation of the mean over bins of 20 measurements in $\theta_{\mathrm{p}}$. The solid line is a fit for all the displayed data; the dotted line is a fit for the data from 3 to 7 June 2000; the dashed line is a fit for data from 8 to 12 June 2000. 


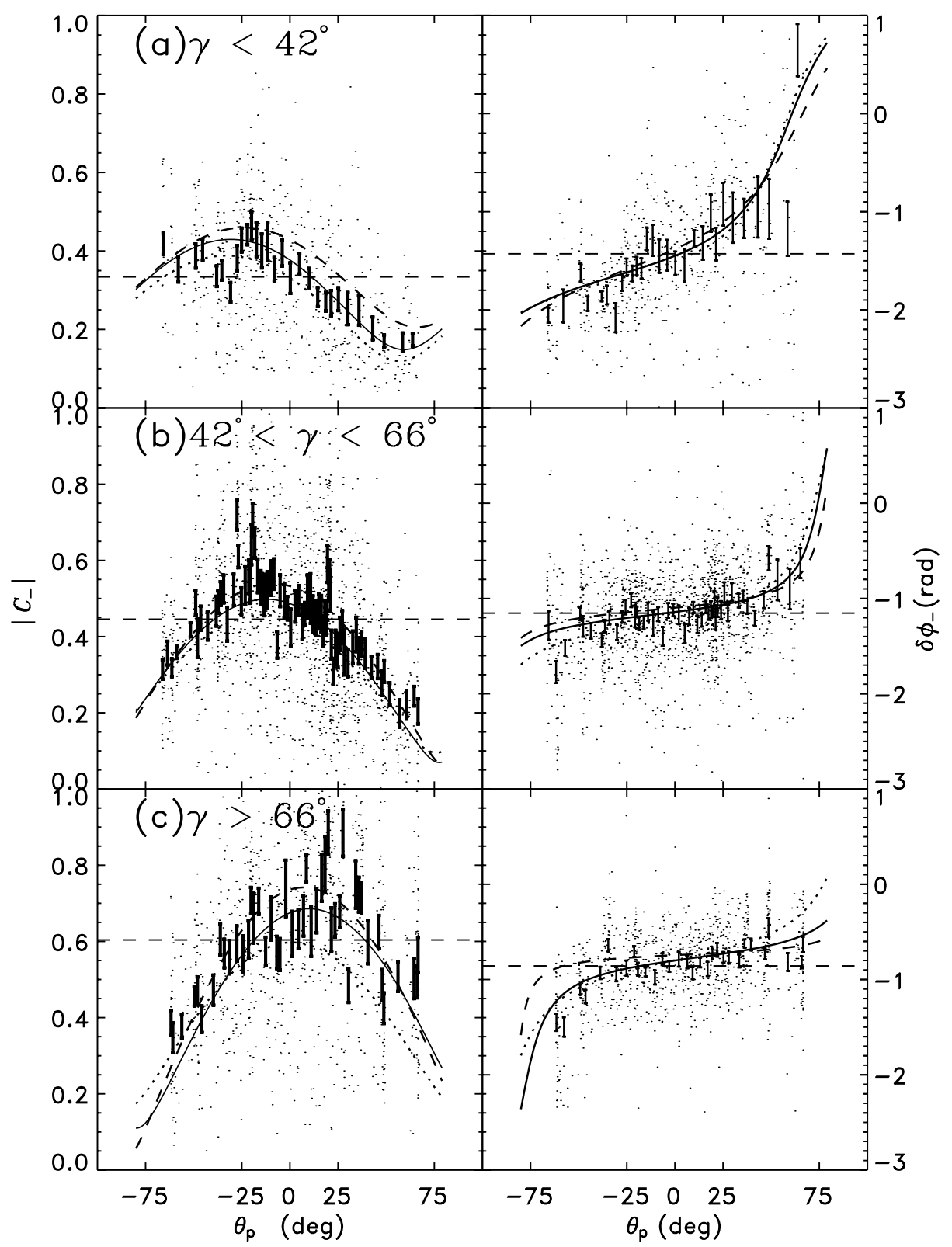

Figure 4 The modulus of the correlation, $\left|C_{-}\right|$(left column), and the phase $\delta \phi_{-}$(right column) in the penumbra of AR9026 at $5 \mathrm{mHz}$ for all days of observation plotted against projected angle $\theta_{\mathrm{p}}$ for different values of magnetic field inclinations as indicated: (a) $\gamma<42^{\circ}$, where the mean field strength is $\langle\mathbf{B}\rangle=1900 \mathrm{G}$; (b) $42^{\circ}<\gamma<66^{\circ}$, where $\langle\mathbf{B}\rangle=1400 \mathrm{G}$; (c) $\gamma>66^{\circ}$, where $\langle\mathbf{B}\rangle=600 \mathrm{G}$. The horizontal dashed lines indicate the mean value of $\left|C_{-}\right|$for each panel. The error bars indicate the standard deviation of the mean over bins of 20 measurements in $\theta_{\mathrm{p}}$. The solid line is a fit for all the displayed data; the dotted line is a fit for the data from 3 to 7 June 2000; the dashed line is a fit for data from 8 to 12 June 2000. 


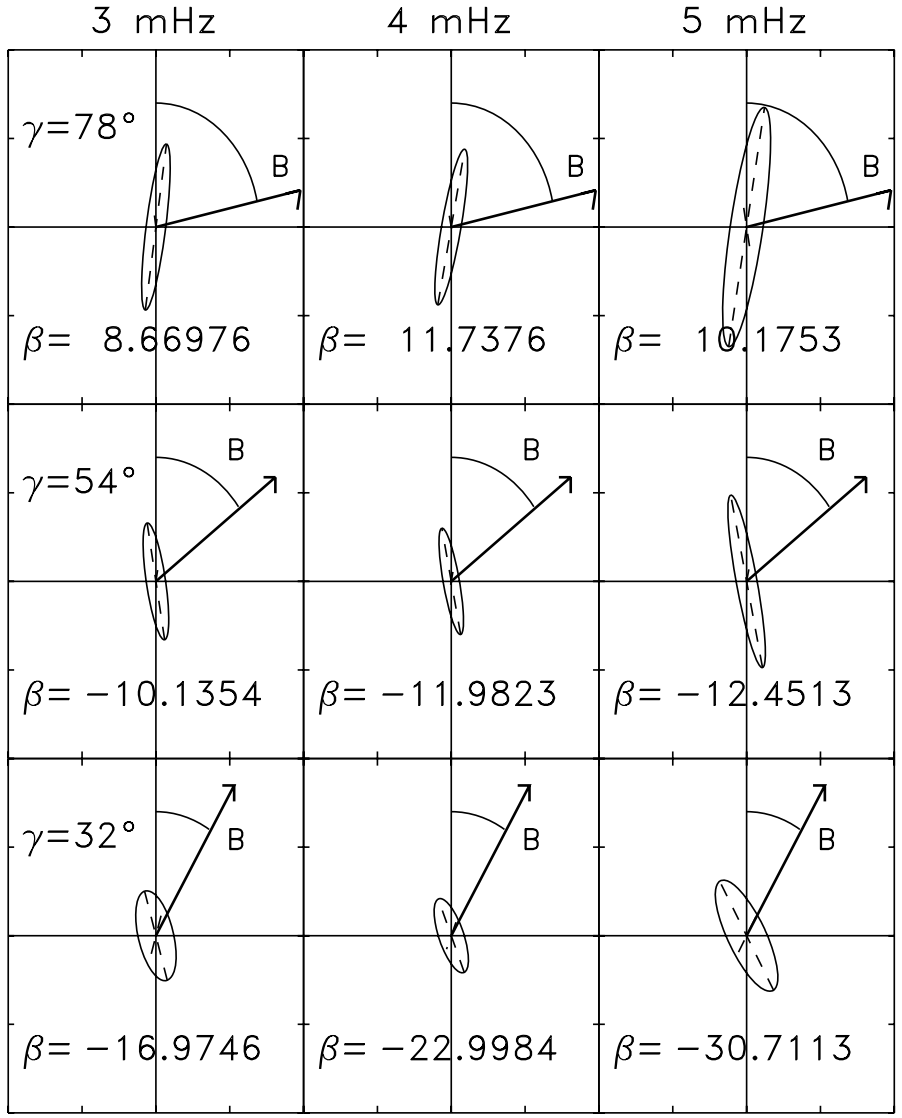

Figure 5 Least-squares-fit surface velocity ellipses as given by the phase and amplitude of the local ingression control correlation in the penumbra of AR9026 for $\gamma>66^{\circ}$ (top row), $42^{\circ}<\gamma<66^{\circ}$ (middle row), and $\gamma<42^{\circ}$ (bottom row). The $\gamma$ listed in the plot is the angle at which the magnetic field vector is drawn. $\beta$ is the inclination angle of the semi-major axis of the velocity ellipse. The left column is for frequencies of $3 \mathrm{mHz}$, the middle column for $4 \mathrm{mHz}$, and the right column for $5 \mathrm{mHz}$.

semi-major axis. We perform a least-squares fit to the observed $\left|C_{-}\right|$and $\delta \phi_{-}$to determine the elliptical motion consistent with the data. The best-fit ellipses are shown in Figures 5 and 9. The moduli and phase of $C_{-}$as determined from the fits are plotted (as solid lines) with the data points in Figures $2-4$ and 6-8. Separate fits were performed for independent five-day subsets of the data (also shown in the figures by the dotted and dashed lines). There are no obvious, systematic differences in the fits over time. The orientations and eccentricities are highly consistent across all frequency bands in both sunspots (Figures 5 and 9). Some systematic differences between the two spots are evident. For AR9026, the ellipses are aligned slightly toward the magnetic field direction at high field inclination but "swing" over as the inclination becomes smaller (and the magnetic field stronger). For AR9057, the motion is nearly vertical at low field inclination, but it also tilts away from the field in the stronger, more vertical fields. In both spots, the eccentricity of the ellipses increases with decreasing field strength or increasing inclination.

We can define a deviation angle as the angle between the magnetic field vector and surface velocity ellipse semi-major axis $(\delta=\gamma-\beta$, where $\beta$ is the inclination of the semi- 


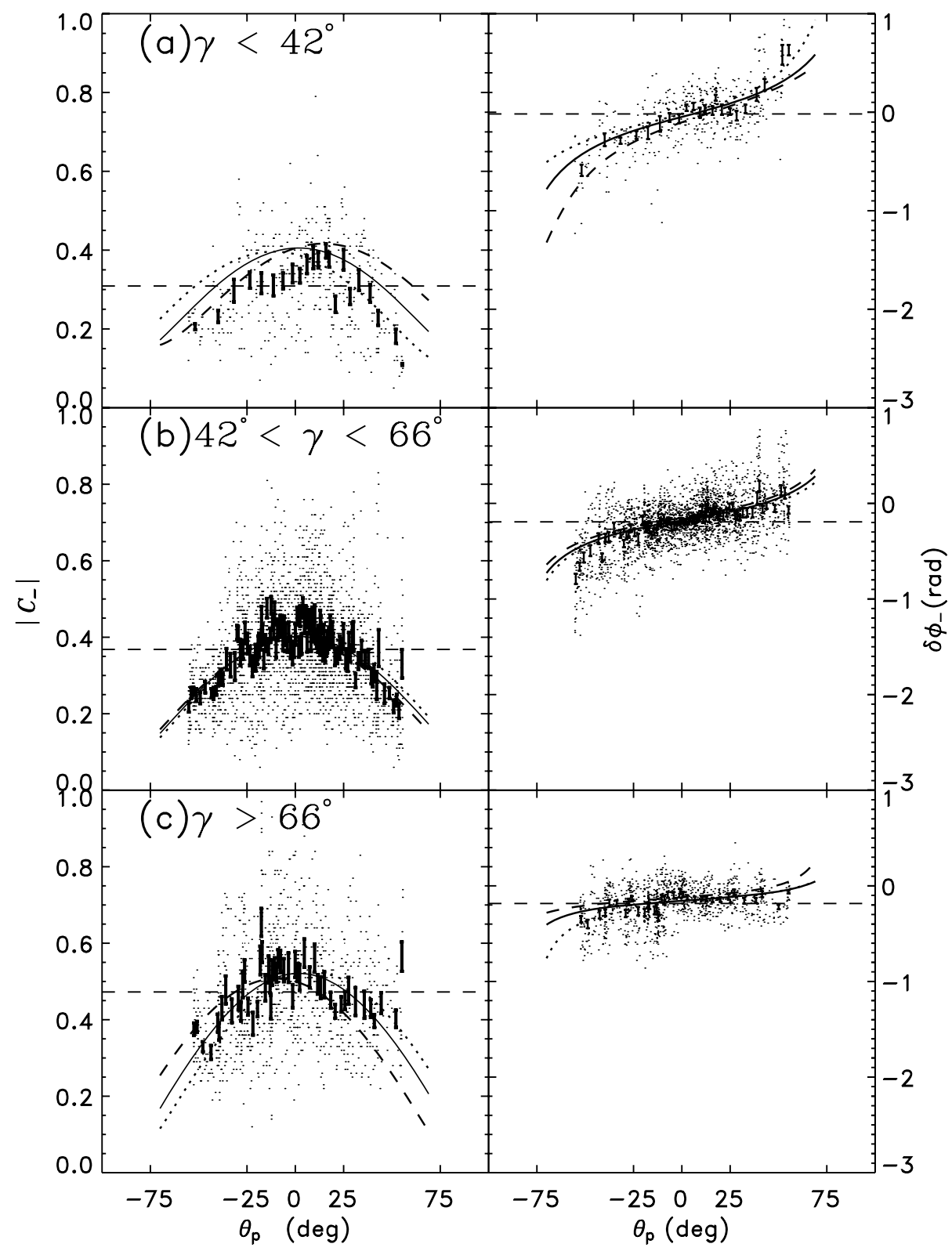

Figure 6 The modulus of the correlation, $\left|C_{-}\right|$(left column), and phase $\delta \phi_{-}$(right column) in the penumbra of AR9057 at $3 \mathrm{mHz}$ for all days of observation plotted against projected angle $\theta_{\mathrm{p}}$ for different values of magnetic field inclinations as indicated: (a) $\gamma<42^{\circ}$, where the mean field strength is $\langle\mathbf{B}\rangle=1700 \mathrm{G}$; (b) $42^{\circ}<\gamma<66^{\circ}$, where $\langle\mathbf{B}\rangle=1000 \mathrm{G}$; (c) $\gamma>66^{\circ}$, where $\langle\mathbf{B}\rangle=600 \mathrm{G}$. The horizontal dashed lines indicate the mean value of $\left|C_{-}\right|$for each panel. The error bars indicate the standard deviation of the mean over bins of 20 measurements in $\theta_{\mathrm{p}}$. The solid line is a fit for all the displayed data; the dotted line is a fit for the data from 24 to 28 June 2000; the dashed line is a fit for data from 29 June to 2 July 2000. 


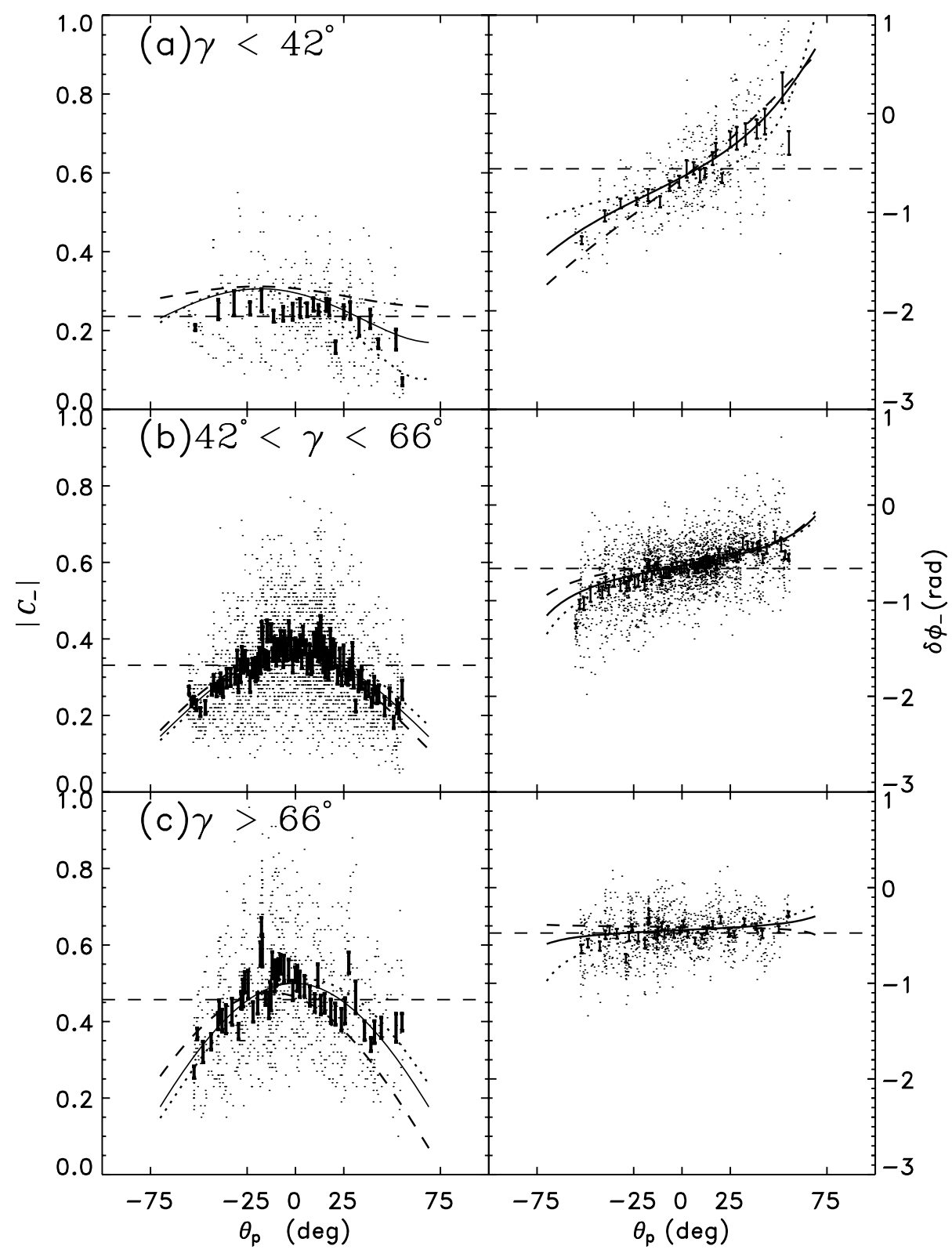

Figure 7 The modulus of the correlation, $\left|C_{-}\right|$(left column), and phase $\delta \phi_{-}$(right column) in the penumbra of AR9057 at $4 \mathrm{mHz}$ for all days of observation plotted against projected angle $\theta_{\mathrm{p}}$ for different values of magnetic field inclinations as indicated: (a) $\gamma<42^{\circ}$, where the mean field strength is $\langle\mathbf{B}\rangle=1700 \mathrm{G}$; (b) $42^{\circ}<\gamma<66^{\circ}$, where $\langle\mathbf{B}\rangle=1000 \mathrm{G}$; (c) $\gamma>66^{\circ}$, where $\langle\mathbf{B}\rangle=600 \mathrm{G}$. The horizontal dashed lines indicate the mean value of $\left|C_{-}\right|$for each panel. The error bars indicate the standard deviation of the mean over bins of 20 measurements in $\theta_{\mathrm{p}}$. The solid line is a fit for all the displayed data; the dotted line is a fit for the data from 24 to 28 June 2000; the dashed line is a fit for data from 29 June to 2 July 2000. 


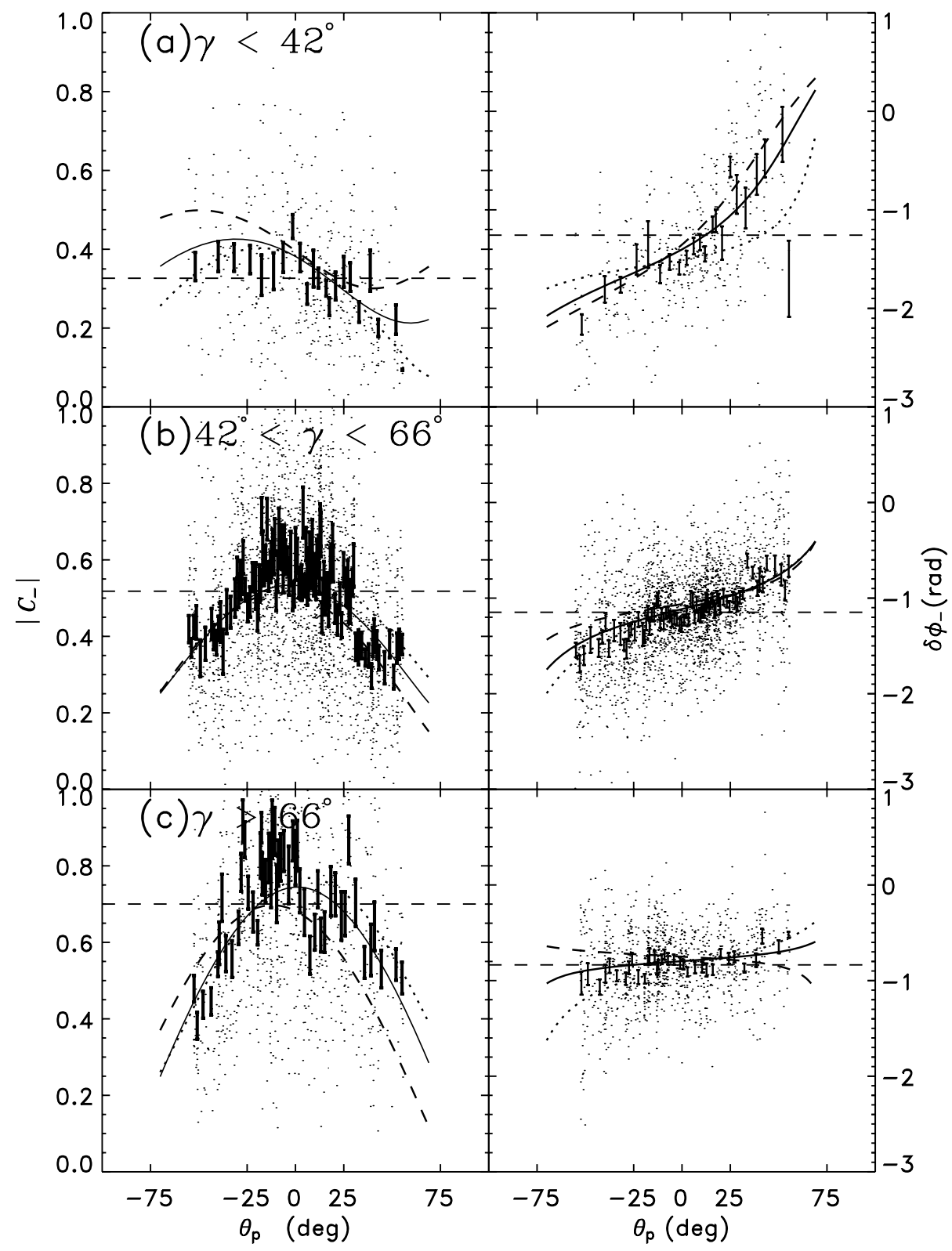

Figure 8 The modulus of the correlation, $\left|C_{-}\right|$(left column), and phase $\delta \phi_{-}$(right column) in the penumbra of AR9057 at $5 \mathrm{mHz}$ for all days of observation plotted against projected angle $\theta_{\mathrm{p}}$ for different values of magnetic field inclinations as indicated: (a) $\gamma<42^{\circ}$, where the mean field strength is $\langle\mathbf{B}\rangle=1700 \mathrm{G}$; (b) $42^{\circ}<\gamma<66^{\circ}$, where $\langle\mathbf{B}\rangle=1000 \mathrm{G}$; (c) $\gamma>66^{\circ}$, where $\langle\mathbf{B}\rangle=600 \mathrm{G}$. The horizontal dashed lines indicate the mean value of $\left|C_{-}\right|$for each panel. The error bars indicate the standard deviation of the mean over bins of 20 measurements in $\theta_{\mathrm{p}}$. The solid line is a fit for all the displayed data; the dotted line is a fit for the data from 24 to 28 June 2000; the dashed line is a fit for data from 29 June to 2 July 2000. 


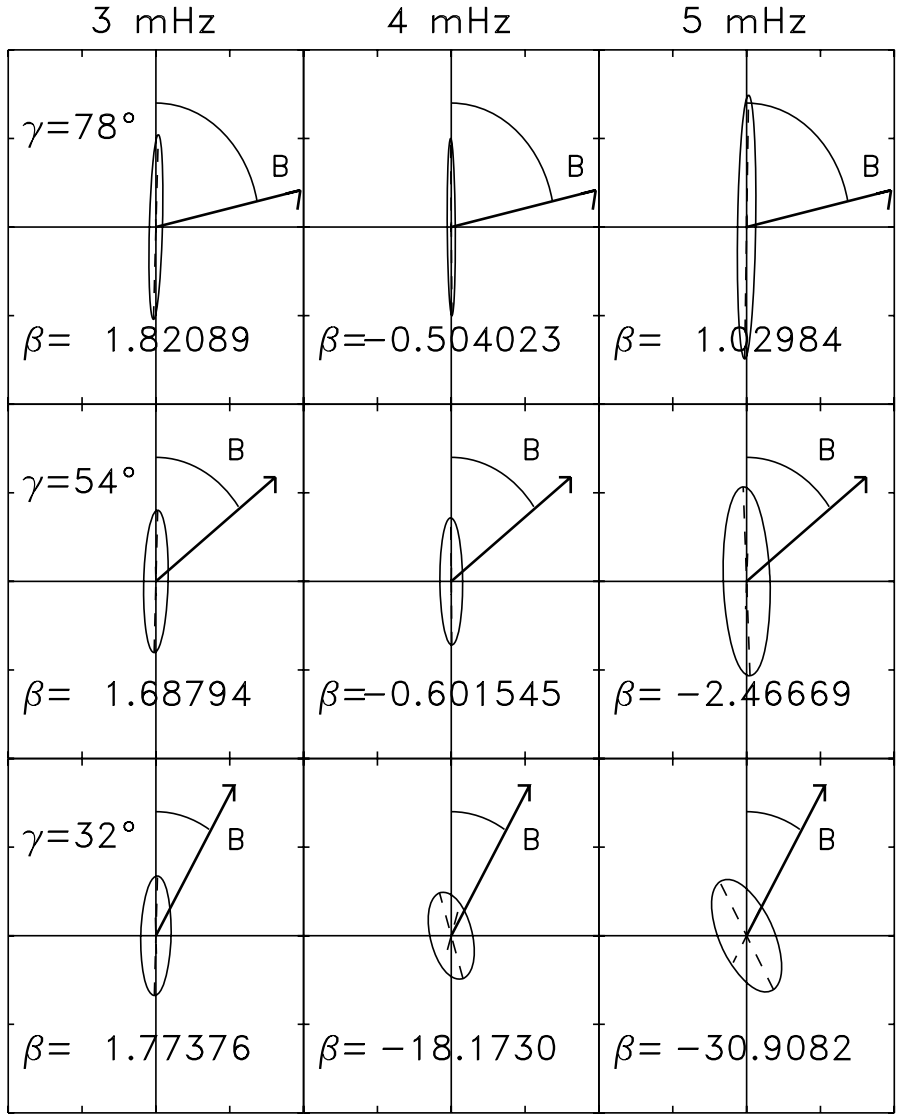

Figure 9 Least-squares-fit surface velocity ellipses as given by the phase and amplitude of the local ingression control correlation in the penumbra of AR9057 for $\gamma>66^{\circ}$ (top row), $42^{\circ}<\gamma<66^{\circ}$ (middle row), and $\gamma<42^{\circ}$ (bottom row). The $\gamma$ listed in the plot is the angle at which the magnetic field vector is drawn. $\beta$ is the inclination angle of the semi-major axis of the velocity ellipse. The left column is for frequencies of $3 \mathrm{mHz}$, the middle column for $4 \mathrm{mHz}$, and the right column for $5 \mathrm{mHz}$.

major axis from the vertical). We show the variation of the deviation angle, length of the semi-major axis, and eccentricity with magnetic-field strength and/or inclination in Figure 10. Also shown is the phase difference between the fits at $\theta_{\mathrm{p}}=-60^{\circ}$ and $\theta_{\mathrm{p}}=+60^{\circ}$. This quantity is generally inversely correlated to the ellipse eccentricity, but it is a more direct measure of the total variation of observed phase shift for a specific penumbral region (Schunker et al., 2005). In Figure 10, data from AR9026 are represented by an asterisk and AR9057 by a diamond, and the frequencies are color-coded as follows: $5 \mathrm{mHz}$ is black, $4 \mathrm{mHz}$ is purple, and $3 \mathrm{mHz}$ is red.

In general, the trends shown in Figure 10 - namely an increase in the deviation angle, eccentricity, and semi-major axis length and a decrease in the phase variation - with increasing inclination are observed in both sunspots and at all frequencies. There are some deviations from this. For example, at low inclinations it is observed that the eccentricity (phase variation) decreases (increases) with frequency. Note that the semi-major-axis length is an indication of the total wave amplitude in the magnetic region. The trend observed in 

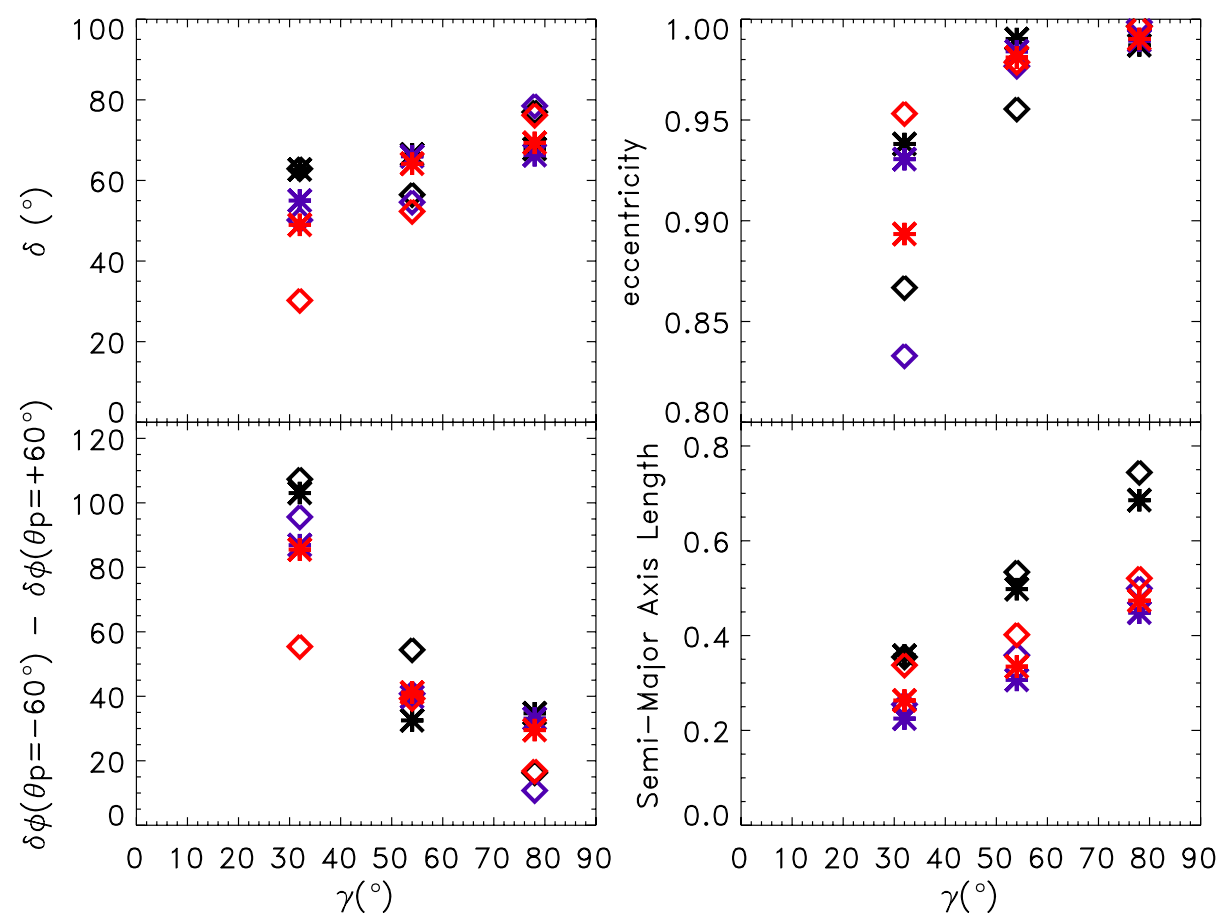

Figure 10 Properties of the ellipses: deviation angle $\delta$ (top left); the ellipse eccentricity for both sunspots at all frequencies against the three average magnetic field inclinations (top right); the phase difference between the least-squares-fit correlation phase at $\theta_{\mathrm{p}}=-60^{\circ}$ and at $\theta_{\mathrm{p}}=+60^{\circ}\left(\delta \phi_{\left(\theta_{\mathrm{p}}=-60^{\circ}\right)}-\delta \phi_{\left(\theta_{\mathrm{p}}=+60^{\circ}\right)}\right)($ bottom left); the length of the semi-major axis of each ellipse in each region for both sunspots at all frequencies against the three average magnetic field inclinations (bottom right). AR9026 is represented by the asterisk and AR9057 by the diamond, and the colors indicate the following frequencies: $5 \mathrm{mHz}$ (black), $4 \mathrm{mHz}$ (purple), and $3 \mathrm{mHz}$ (red).

the lower left panel of Figure 10 is consistent with a reduction in wave amplitude related to the field strength.

\section{Local Egression Control Correlation}

In this section we examine the phase of the local egression control correlation,

$$
C_{+}(\mathbf{r}, v)=\left\langle\left.\hat{H}_{+}(\mathbf{r}, v) \hat{\psi}^{*}(\mathbf{r}, v)\right|_{\Delta v}=\left|C_{+}\right| \mathrm{e}^{-\mathrm{i} \delta \phi_{+}} .\right.
$$

Since the egression is simply the time reverse of the ingression, we might expect to see a reversal of the phase change compared to the ingression phases.

Using all of the days of data, we plot the egression correlation phase against $\theta_{\mathrm{p}}$ in Figures 11 and 12, along with the fits for the phase variation caused by elliptical motion. We see similar trends in both AR9026 and AR9787. Figure 13 shows the phase difference between the least-squares-fit correlation phase at $\theta_{\mathrm{p}}=-60^{\circ}$ and at $\theta_{\mathrm{p}}=+60^{\circ}$ of the ingression plotted against that of the egression. The colors and symbols represent the same as before: AR9026 is represented by an asterisk and AR9057 by a diamond, and the frequencies are 


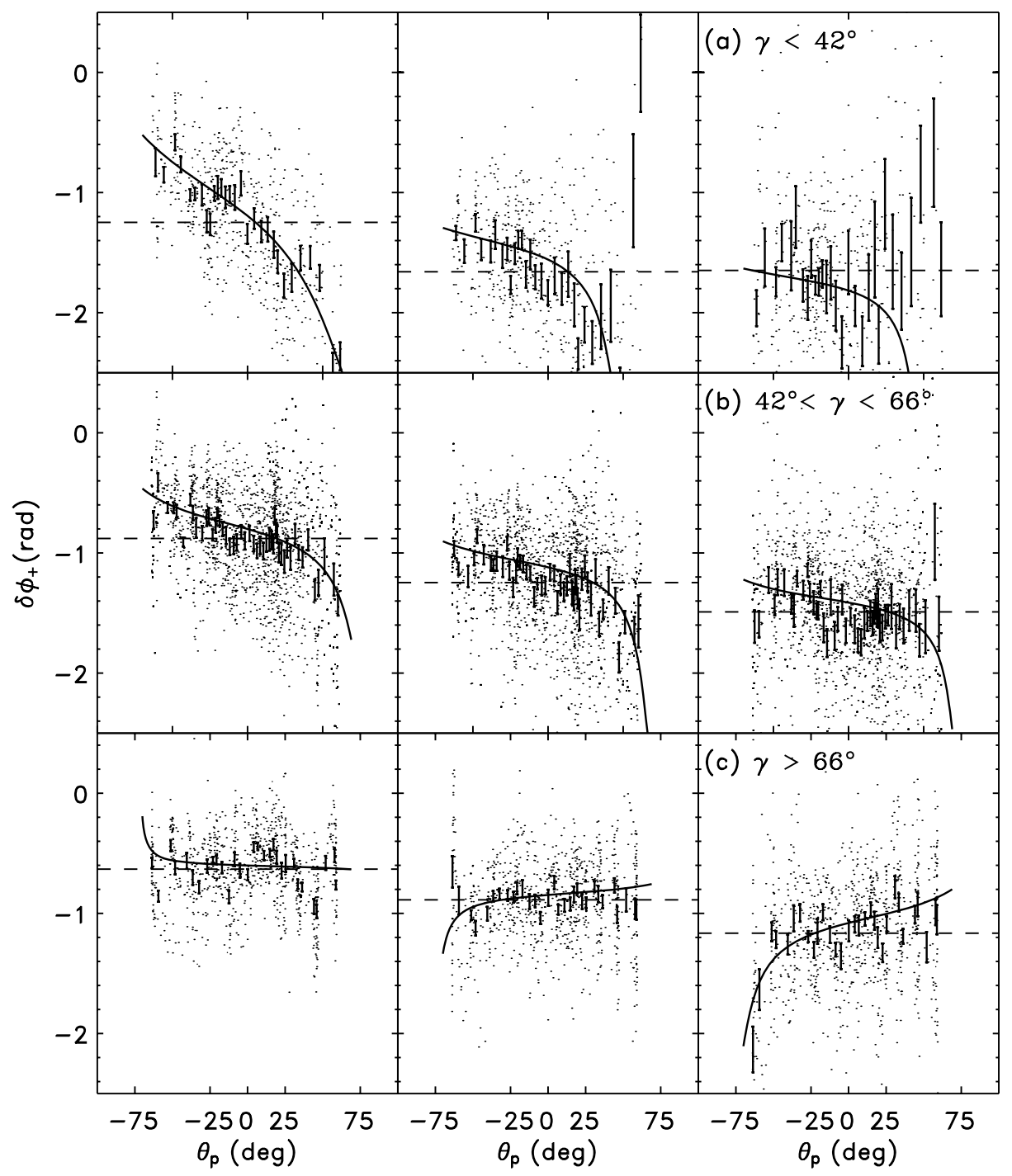

Figure 11 The 3-mHz (left column), 4-mHz (middle column), and 5-mHz (right column) egression correlation phase $\left(\delta \phi_{+}\right)$versus $\theta_{\mathrm{p}}$ within the penumbra of sunspot AR9026 for different values of magnetic field inclination as indicated: (a) $\gamma<42^{\circ}$, where the mean field strength is $\langle\mathbf{B}\rangle=1900 \mathrm{G}$; (b) $42^{\circ}<\gamma<66^{\circ}$, where $\langle\mathbf{B}\rangle=1400 \mathrm{G}$; (c) $\gamma>66^{\circ}$, where $\langle\mathbf{B}\rangle=600 \mathrm{G}$. The three rows represent different portions of the penumbra as shown in Figure 1 (bottom). The horizontal dashed lines indicate the mean value of $\delta \phi_{+}$for each panel.

$5 \mathrm{mHz}$ (black), $4 \mathrm{mHz}$ (purple), and $3 \mathrm{mHz}$ (red); in addition the size of the symbols represent the average inclination from vertical. The solid line has a slope of -1 . There is a reverse behavior of the ingression, compared to the previous egression results, present for all frequencies at most magnetic field inclinations. The exception is when the field is highly inclined, where we observe a trend in the same sense as the ingression. This is unexpected and warrants further study. 


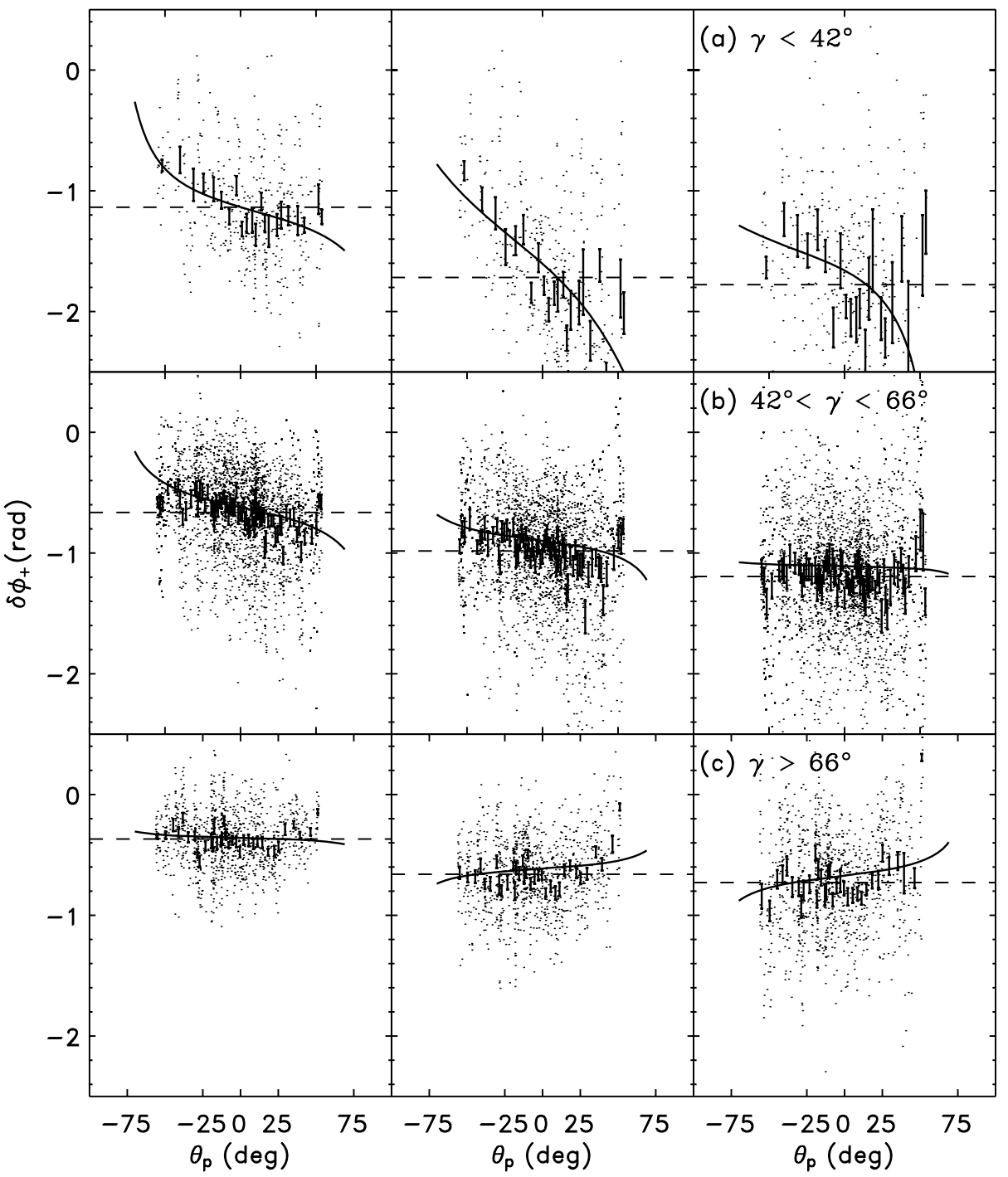

Figure 12 The 3-mHz (left column), 4-mHz (middle column), and $5 \mathrm{mHz}$ (right column) egression correlation phase $\left(\delta \phi_{+}\right)$versus $\theta_{\mathrm{p}}$ within the penumbra of sunspot AR9057 for different values of magnetic field strength as indicated: (a) $\gamma<42^{\circ}$, where the mean field strength is $\langle\mathbf{B}\rangle=1700 \mathrm{G}$; (b) $42^{\circ}<\gamma<66^{\circ}$, where $\langle\mathbf{B}\rangle=1000 \mathrm{G}$; (c) $\gamma>66^{\circ}$, where $\langle\mathbf{B}\rangle=600 \mathrm{G}$. The three different panels represent different portions of the penumbra, similar to Figure 1 (top). The horizontal dashed lines indicate the mean value of $\delta \phi_{+}$for each panel.

\section{Discussion}

Two-dimensional mode conversion predicts (among other things; Cally, 2007) that when the attack angle is small most of the observable energy will be in the slow acoustic mode and when the attack angle is large most of the observable energy will be in the fast magnetic mode. In this case we will be seeing the line-of-sight effect of a combination of waves coming from all directions impinging on magnetic field with a particular orientation. The ob- 


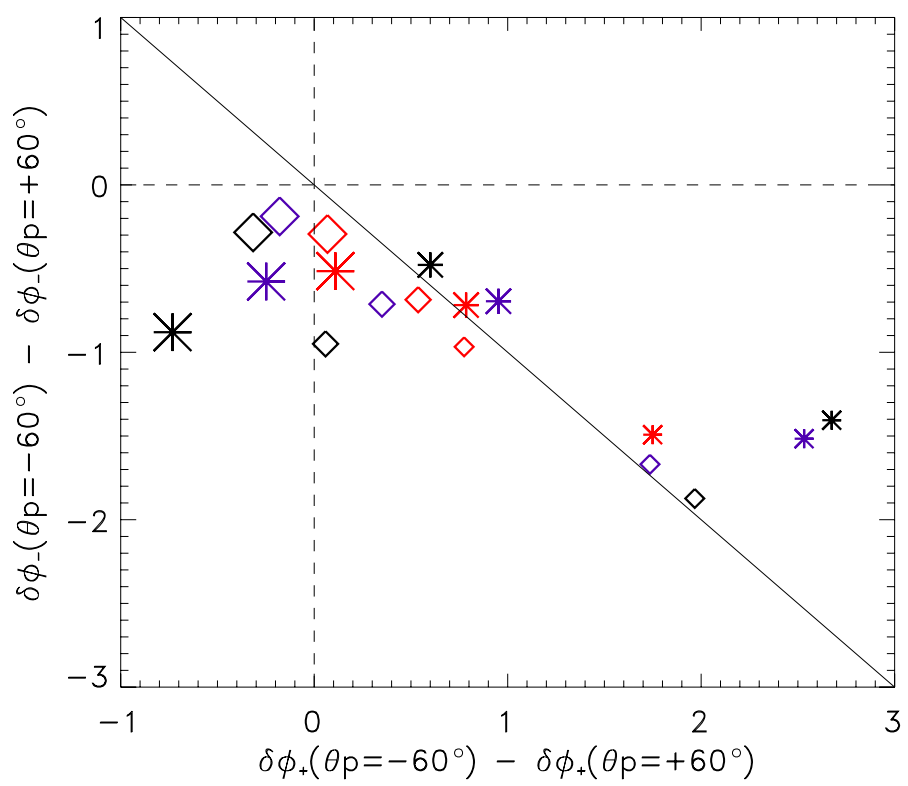

Figure 13 The phase difference between the least-squares-fit correlation phase at $\theta_{\mathrm{p}}=-60^{\circ}$ and at $\theta_{\mathrm{p}}=+60^{\circ}$ of the ingression plotted against that of the egression. The solid line is a line of slope -1 . AR9026 is represented by an asterisk and AR9057 by a diamond, and the colors indicate the following frequencies: $5 \mathrm{mHz}$ (black), $4 \mathrm{mHz}$ (purple), and $3 \mathrm{mHz}$ (red). The largest symbols represent $\gamma>66^{\circ}$ and the smallest symbols $\gamma<42^{\circ}$.

servations by MDI consist of line-of-sight Doppler signatures of the surface motion, which are presumably caused mainly by pressure perturbations. This means that we would expect to be observing only the slow acoustic mode; however, it is possible that we are observing a combination of the acoustic and magnetic modes.

The dependence of the phase shift of the observed ingression correlations with azimuthal angle around sunspot penumbrae, as viewed from different observational vantage points, shows that the incoming phase shifts must be (at least partly) photospheric in origin and are influenced by the presence of inclined magnetic fields (Schunker et al., 2005). Analysis of the variation of both the amplitude and phase of the surface velocities provides an opportunity to characterize the magnetically influenced acoustic signature as an ellipse with properties determined by the magnetic fields. We found that the ellipses are either nearly vertical (for weaker, more inclined fields) or generally directed away from the magnetic field direction (for stronger, more vertical fields). Largely consistent results for the two active regions AR9026 and AR9057 are found. Some properties of the surface ellipses (e.g., their inclinations) are different for the two sunspots. Some of this variation may be due to differences in the field properties. For example, the field in AR9057 is on average $\approx 15 \%$ weaker than that in AR9026.

Fits of the elliptical motion in Figures 5 and 9 depend critically on the correlation modulus, which is prone to systematic uncertainties. But the trend is that a stronger, less inclined magnetic field produces elliptical motion with smaller amplitude, eccentricity, and deviation angle and a larger inclination from the vertical. These trends exist for both spots and, by and large, at all frequencies. The shorter semi-major axis at strong magnetic field strengths is consistent with previous knowledge of surface acoustic amplitude suppression in magnetic 
fields. It is curious to note, however, that at $4 \mathrm{mHz}$ the amplitude is consistently smaller than at $3 \mathrm{mHz}$.

These are the first results to estimate the behavior of the surface velocity ellipse at the photosphere within sunspots. The results do not immediately suggest an observation of the slow wave as shown by Cally (2005) or Schunker and Cally (2006), but they are consistent with their expectations of the behavior of slow waves at this height in the atmosphere. Mode conversion theory states that the alignment depends on $a^{2} / c^{2}$, which at the observational heights of $\approx 200 \mathrm{~km}$ in the atmosphere may not be large enough to invoke alignment. Since the ray analysis is somewhat unrealistic, we would expect to observe a combination of fast and slow waves at the surface, which will contribute to a spurious view of the surface velocities. In three dimensions, where the wave vector is not in the vertical plane containing the magnetic field lines, Cally and Goossens (2007) have shown theoretically that there should also be substantial conversion to an upgoing Alfvén wave, which further complicates any analysis of oscillation modes in the low solar magnetic atmosphere. Simultaneous multiheight observations would be useful in resolving this issue. Our results are necessary, but not sufficient, for conclusive evidence of mode conversion occurring close to the solar surface. Nevertheless, at this stage, there is nothing in our results that is inconsistent with MHD mode conversion.

Rajaguru et al. (2007) are currently exploring the possibility that these apparent surface effects are due to changes in radiative transfer within active regions and the formation height of the observational $\mathrm{Ni} 678 \mathrm{~nm}$ line. This explanation requires an absorption mechanism or some other means of producing a difference between the amplitudes of upward and downward propagating waves. Thus mode conversion may still be important in this proposed mechanism. A test of the mechanism proposed by Rajaguru et al. (2007) would be to repeat the observations performed here in a magnetically insensitive line, where the proposed radiative transfer effects would not be present. In terms of mode conversion, it is suggested that the main effect occurs along the bright radial filaments of the interlocking comb structure as presented in the penumbral models of Weiss et al. (2004). However, observational helioseismic spatial resolution cannot currently resolve this.

This is also the first time that the variation of the phase of the local egression correlation has been analyzed in the penumbra. It is curious that the egression correlation shows a reverse dependence when the magnetic field is weak and highly inclined. This is evidence of a reverse ingression dependence on the line of sight, but further investigation is required to understand the behavior at high frequencies in the weaker, more inclined fields.

Open Access This article is distributed under the terms of the Creative Commons Attribution Noncommercial License which permits any noncommercial use, distribution, and reproduction in any medium, provided the original author(s) and source are credited.

\section{References}

Basu, S., Antia, H.M., Bogart, R.S.: 2004, Ring-diagram analysis of the structure of solar active regions. Astrophys. J. 610, 1157.

Braun, D.C.: 1995, Scattering of p-modes by sunspots. I. Observations. Astrophys. J. 451, 859.

Braun, D.C.: 1997, Time-distance sunspot seismology with GONG data. Astrophys. J. 487, 447.

Braun, D.C., Lindsey, C.: 2000, Phase-sensitive holography of Solar activity. Solar Phys. $192,307$.

Cally, P.S.: 2005, Local magnetohelioseismology of active regions. Mon. Not. Roy. Astron. Soc. $358,353$.

Cally, P.S.: 2007, What to look for in the seismology of solar active regions. Astron. Nachr. 328, 286.

Cally, P.S., Goossens, M.: 2007, Three-dimensional MHD wave propagation and conversion to Alfvén waves near the Solar surface. I. Direct numerical solution. Solar Phys. doi:10.1007/s11207-007-9086-3. 
Cally, P.S., Crouch, A.D., Braun, D.C.: 2003, Probing sunspot magnetic fields with $p$-mode absorption and phase shift data. Mon. Not. Roy. Astron. Soc. 346, 381.

Couvidat, S., Rajaguru, S.P.: 2007, Contamination by surface effects of time-distance helioseismic inversions for sound speed beneath sunspots. Astrophys. J. 661, 558.

Couvidat, S., Birch, A.C., Kosovichev, A.G.: 2006, Three-dimensional inversion of sound speed below a sunspot in the Born approximation. Astrophys. J. 640, 516.

Crouch, A., Cally, P.S.: 2003, Mode conversion of solar $p$ modes in non-vertical magnetic fields. I. Twodimensional model. Solar Phys. 214, 201.

Duvall, T.L., Jr., Jefferies, S.M., Harvey, J.W., Pomerantz, M.A.: 1993, Time-distance helioseismology. Nature 362, 430.

Hanasoge, S.M., Couvidat, S., Rajaguru, S.P., Birch, A.C.: 2007, Impact of locally suppressed wave sources on helioseismic travel times. 707.

Korzennik, S.G.: 2006, The cookie cutter test for time-distance tomography of active regions. In: Fletcher, K., Thompson, M. (eds.) Proceedings of SOHO 18/GONG 2006/HELAS I, Beyond the Spherical Sun 624, ESA, Noordwijk, 60.

Kosovichev, A.G., Duvall, T.L.J., Scherrer, P.H.: 2000, Time-distance inversion methods and results. Solar Phys. 192, 159.

Lindsey, C., Braun, D.C.: 2000, Basic principles of Solar acoustic holography. Solar Phys. 192, 261.

Lindsey, C., Braun, D.C.: 2005a, The acoustic showerglass. I. Seismic diagnostics of photospheric magnetic fields. Astrophys. J. 620, 1107.

Lindsey, C., Braun, D.C.: 2005b, The acoustic showerglass. II. Imaging active region subphotospheres. Astrophys. J. 620, 1118.

Mickey, D.L., Canfield, R.C., Labonte, B.J., Leka, K.D., Waterson, M.F., Weber, H.M.: 1996, The imaging vector magnetograph at Haleakala. Solar Phys. 168, 229.

Rajaguru, S.P., Birch, A.C., Duvall, T.L., Jr., Thompson, M.J., Zhao, J.: 2006, Sensitivity of time-distance helioseismic measurements to spatial variation of oscillation amplitudes. I. Observations and a numerical model. Astrophys. J. 646, 543.

Rajaguru, S.P., Sankarasubramanian, K., Wachter, R., Scherrer, P.H.: 2007, Radiative transfer effects on Doppler measurements as sources of surface effects in sunspot seismology. Astrophys. J. 654, L175.

Scherrer, P.H., Bogart, R.S., Bush, R.I., Hoeksema, J.T., Kosovichev, A.G., Schou, J., Rosenberg, W., Springer, L., Tarbell, T.D., Title, A., Wolfson, C.J., Zayer, I., MDI Engineering Team, 1995, The Solar oscillations investigation - Michelson Doppler imager. Solar Phys. 162, 129.

Schunker, H., Cally, P.S.: 2006, Magnetic field inclination and atmospheric oscillations above solar active regions. Mon. Not. Roy. Astron. Soc. 372, 551.

Schunker, H., Braun, D.C., Cally, P.S.: 2007, Surface magnetic field effects in local helioseismology. Astron. Nachr. 328, 292.

Schunker, H., Braun, D.C., Cally, P.S., Lindsey, C.: 2005, The local helioseismology of inclined magnetic fields and the showerglass effect. Astrophys. J. 621, L149.

Weiss, N.O., Thomas, J.H., Brummell, N.H., Tobias, S.M.: 2004, The origin of penumbral structure in sunspots: Downward pumping of magnetic flux. Astrophys. J. 600, 1073.

Zhao, J., Kosovichev, A.G.: 2003, Helioseismic observation of the structure and dynamics of a rotating sunspot beneath the solar surface. Astrophys. J. 591, 446.

Zhao, J., Kosovichev, A.G.: 2006, Surface magnetism effects in time-distance helioseismology. Astrophys. J. 643, 1317. 